\title{
THE GEMINI SPECTRAL LIBRARY OF NEAR-IR LATE-TYPE STELLAR TEMPLATES AND ITS APPLICATION FOR VELOCITY DISPERSION MEASUREMENTS*
}

\author{
Cláudia Winge ${ }^{1}$, Rogemar A. Riffel ${ }^{2}$, and Thaisa Storchi-Bergmann ${ }^{2}$ \\ ${ }^{1}$ Gemini Observatory, c/o Aura, Inc., Casilla 603, La Serena, Chile; cwinge@ gemini.edu \\ ${ }^{2}$ Universidade Federal do Rio Grande do Sul, IF, CP 15051, Porto Alegre 91501-970, RS, Brazil \\ Received 2009 July 14; accepted 2009 October 9; published 2009 October 29
}

\begin{abstract}
We present a spectroscopic library of late spectral type stellar templates in the near-IR range $2.15-2.42 \mu \mathrm{m}$, at $R=$ 5300-5900 resolution, oriented to support stellar kinematics studies in external galaxies, such as the direct determination of the masses of supermassive black holes in nearby active (or non-active) galaxies. The combination of high spectral resolution and state-of-the-art instrumentation available in $8 \mathrm{~m}$ class telescopes has made the analysis of circumnuclear stellar kinematics using the near-IR CO band heads one of the most used techniques for such studies, and this library aims to provide the supporting data sets required by the higher spectral resolution and larger spectral coverage currently achieved with modern near-IR spectrographs. Examples of the application for kinematical analysis are given for data obtained with two Gemini instruments, but the templates can be easily adjusted for use with other near-IR spectrographs at similar or lower resolution. The example data sets are also used to revisit the "template mismatch" effect and the dependence of the velocity dispersion values obtained from the fitting process with the characteristics of the stellar templates. The library is available in electronic form from the Gemini Web pages.
\end{abstract}

Key words: astronomical data bases: miscellaneous - methods: data analysis - stars: late-type - techniques: spectroscopic

\section{INTRODUCTION}

Spectral templates, usually late-type stars, are required for the analysis of kinematical data on external galaxies or other stellar ensembles (e.g., Emsellem et al. 2001; Márquez et al. 2003; Barbosa et al. 2006; Ganda et al. 2006; Cappellari et al. 2007; Dumas et al. 2007; Riffel et al. 2008; Cappellari et al. 2009; Riffel et al. 2009). In the near-infrared (hereafter near-IR), the most commonly used features are the $\mathrm{CO}$ overtone bands at $\lambda>2.29 \mu \mathrm{m}$. These band heads originate from evolved stars and are the strongest features in the $1-3 \mu \mathrm{m}$ spectral range of stellar systems. The features are deep and sharp, and at least the first two overtones are located in regions of the IR spectrum relatively clean from telluric lines.

Although observational and theoretical libraries exist at lower spectral resolutions $(R \leqslant 3000$; e.g., Kleinmann \& Hall 1986; Wallace \& Hinkle 1997; Ramirez et al. 1997; Förster Schreiber 2000; Ivanov et al. 2004), no comprehensive set of stellar kinematic templates was available for use with the $R \sim 6000$ configuration of the two Gemini NIR instruments used for stellar population kinematic studies in external galaxies, the Nearinfrared Integral Field Spectrograph (NIFS) and the Gemini Near-Infrared Spectrograph (GNIRS) with the 111 line $\mathrm{mm}^{-1}$ grating (both long-slit and Integral Field Unit-IFU), and, therefore, all observing programs using those configurations would invariably spend some science time taking a small set of stellar spectra to use as templates. This led to a constant multiplication of data taking, since those targets were defined as program calibrations and were not made available to other users until the end of the default 18-month proprietary period.

During semester 2006B at Gemini South, given the unusually poor conditions over the whole semester, a Director's Discretionary "poor weather" program was specifically carried out to provide the NIR community with a larger set of late (F7-M3 types) stellar spectra in the range $2.24-2.43 \mu \mathrm{m}$, including the

\footnotetext{
* http://www.gemini.edu/sciops/instruments/nearir-resources/?q=node/10167.
}

four CO overtone bands, at $R \sim 5900$ resolution. Most of the targets were also observed at a slightly bluer spectral range $(2.15-2.33 \mu \mathrm{m})$ to improve usefulness for NIFS users, overlapping with the red setting on the first two CO bands.

To the original sample of 29 stars observed with GNIRS, another 11 were added from NIFS observations obtained as part of programs GN-2006A-SV-123, GN-2006B-Q-107, and GN-2007A-Q-25, covering the full range $2.1-2.5 \mu \mathrm{m}$ at a similar resolution to that of the GNIRS data.

\section{OBSERVATIONS AND DATA REDUCTION}

\subsection{The GNIRS Data}

GNIRS (Elias et al. 1998) is a multi-configuration spectrograph originally deployed at Gemini South, now moved to Gemini North, that allows for several combinations of resolution and wavelength coverage. The two modes most commonly used for the science relevant here were the long-slit with the 32 line $\mathrm{mm}^{-1}$ or 111 line $\mathrm{mm}^{-1}$ gratings, which yield resolutions of up to $R \sim 2000$ and $R \sim 6000$, respectively (depending on the slit used), and the Integral Field Unit with the same gratings, where the internal optics projected each 0". 15 slice into two detector pixels in the dispersion direction, resulting in a single fixed resolution similar to the one obtained with the 0.3 long-slit, and a field of view of $3{ }^{\prime \prime} .2 \times 4{ }^{\prime \prime} .8$. The IFU has been decommissioned at Gemini North, given the capability overlap with NIFS.

The observed sample was selected from a list kindly provided by G. Doppmann, compiled from the literature (mostly based in Cayrel de Strobel et al. 1997), and the selection was based exclusively on observability: targets that were visible for as long as possible during the semester, bright enough to provide the desired $\mathrm{S} / \mathrm{N}$ on a reasonable on-source time under cloudy, poor seeing conditions, and having a hot (A0-A7) star close enough — and bright enough — to be used for telluric correction. In addition, both target and telluric stars had to have 
Table 1

GNIRS Sample

\begin{tabular}{|c|c|c|c|c|c|c|c|c|}
\hline \multirow[t]{2}{*}{ Object } & \multirow{2}{*}{$\begin{array}{l}\text { Mag } \\
(\mathrm{V})\end{array}$} & \multirow[t]{2}{*}{ Sp Type } & \multirow{2}{*}{$\begin{array}{l}T_{\text {eff }} \\
(\mathrm{K})\end{array}$} & \multirow[t]{2}{*}{$\log g$} & \multicolumn{2}{|c|}{ Blue Setting $(2.15-2.33 \mu \mathrm{m})$} & \multicolumn{2}{|c|}{ Red Setting $(2.24-2.43 \mu \mathrm{m})$} \\
\hline & & & & & Date & Res. $(\AA)$ & Date & Res. $(\AA)$ \\
\hline HD 20038 & 8.91 & F7 IIIw & 5196 & 2.62 & $\begin{array}{l}2006 \text { Oct } 13 \\
2006 \text { Oct } 18\end{array}$ & 2.97 & $\begin{array}{l}2006 \text { Sep } 12 \\
2006 \text { Oct } 7 \\
2006 \text { Oct } 13\end{array}$ & 2.76 \\
\hline HD 209750 & 2.90 & $\mathrm{G} 2 \mathrm{Ib}$ & 5091 & 1.45 & .. & $\ldots$ & 2006 Oct 20 & 2.99 \\
\hline HD 6461 & 7.65 & G3 V & 5110 & 2.30 & 2006 Oct 10 & 2.98 & $\begin{array}{l}2006 \text { Sep } 5 \\
2006 \text { Oct } 7\end{array}$ & 2.93 \\
\hline HD 173764 & 4.23 & G4 IIa & 4700 & 0.94 & 2006 Oct 12 & 3.02 & $\begin{array}{l}2006 \text { Sep } 4 \\
2006 \text { Oct } 21\end{array}$ & 2.89 \\
\hline HD 36079 & 2.84 & G5 II & 5170 & 2.27 & 2007 Jan 4 & 3.16 & 2006 Sep 14 & 3.02 \\
\hline HD 1737 & 5.17 & G5 III & 4790 & 3.05 & 2006 Oct 8 & 2.93 & 2006 Sep 14 & 3.02 \\
\hline HD 213789 & 5.89 & G6 III & 4820 & 2.88 & & $\ldots$ & 2006 Sep 4 & 2.89 \\
\hline HD 212320 & 5.92 & G6 III & 4790 & 2.87 & 2006 Oct 6 & 2.94 & 2006 Sep 4 & 2.89 \\
\hline HD 213009 & 3.97 & G7 III & 4800 & 2.0 & $\ldots$ & $\ldots$ & 2006 Sep 3 & 3.10 \\
\hline HD 35369 & 4.14 & G8 III & 4880 & 2.76 & 2006 Oct 15 & 2.96 & 2006 Oct 5 & 2.88 \\
\hline HD 64606 & 7.44 & G8 V & 5040 & 4.0 & 2007 Jan 4 & 3.16 & 2007 Jan 2 & 2.79 \\
\hline HD 224533 & 4.89 & G9 III & 4960 & 3.19 & 2007 Jan 7 & 2.79 & 2006 Sep 14 & 3.02 \\
\hline HD 4188 & 4.78 & K0 III & 4755 & 2.90 & 2006 Oct 12 & 3.02 & 2006 Oct 5 & 2.88 \\
\hline HD 206067 & 5.11 & K0 III & 4740 & 2.73 & 2006 Oct 6 & 2.94 & 2006 Aug 31 & 2.99 \\
\hline HD 34642 & 4.83 & K0 IV & 4730 & 3.39 & 2006 Oct 17 & 2.89 & 2006 Sep 12 & 2.71 \\
\hline HD 198700 & 3.66 & K1 II & 4383 & 0.80 & $\ldots$ & $\ldots$ & $\begin{array}{l}2006 \text { Sep } 4 \\
2006 \text { Oct } 21\end{array}$ & 2.89 \\
\hline HD 218594 & 3.66 & K1 III & 4430 & 2.34 & 2006 Oct 20 & 2.93 & 2006 Sep 3 & 3.10 \\
\hline HD 26965 & 4.41 & $\mathrm{~K} 1 \mathrm{~V}(\mathrm{a})$ & 5091 & 4.31 & 2006 Dec 12 & 3.07 & $\begin{array}{l}2006 \text { Sep } 13 \\
2006 \text { Oct } 1\end{array}$ & 3.01 \\
\hline HD 39425 & 3.12 & K2 III & 4582 & 2.80 & 2006 Dec 12 & 3.07 & 2006 Oct 1 & 3.01 \\
\hline HD 38392 & 6.15 & $\mathrm{~K} 2 \mathrm{~V}$ & 4990 & 4.50 & 2006 Oct 17 & 2.89 & 2006 Oct 1 & 3.01 \\
\hline HD 4730 & 5.61 & K3 III & 4220 & 2.10 & 2006 Oct 8 & 2.93 & 2006 Sep 3 & 3.10 \\
\hline HD 191408 & 5.31 & K3 V & 4893 & 4.55 & $\ldots$ & $\ldots$ & $\begin{array}{l}2006 \text { Sep } 5 \\
2006 \text { Oct } 1\end{array}$ & 3.01 \\
\hline HD 9138 & 4.84 & K4 III & 4040 & 1.91 & 2006 Oct 19 & 2.97 & 2006 Sep 10 & 2.94 \\
\hline HD 720 & 5.42 & K5 III & 4160 & 2.02 & 2006 Oct 12 & 3.02 & 2006 Sep 10 & 2.94 \\
\hline HD $32440^{\mathrm{a}}$ & 5.47 & K6 III & $\ldots$ & $\ldots$ & 2007 Jan 6 & 2.79 & 2007 Jan 4 & 2.73 \\
\hline HD $63425 B^{a}$ & 7.71 & K7 III & $\ldots$ & $\ldots$ & 2006 Dec 12 & 3.07 & 2006 Dec 12 & 2.75 \\
\hline HD $113538^{a, b}$ & 9.02 & $\mathrm{~K} 8 \mathrm{~V}$ & $\ldots$ & $\ldots$ & 2007 Jan 4 & 3.16 & 2007 Jan 6 & 2.90 \\
\hline HD 2490 & 5.43 & M0 III & 3652 & 4.0 & 2006 Oct 15 & 2.96 & 2006 Sep 11 & 2.95 \\
\hline HD 112300 & 3.38 & M3 III & 3652 & 1.3 & $\ldots$ & $\ldots$ & 2007 Jan 16 & 2.89 \\
\hline
\end{tabular}

Notes. The spectral resolution in columns (7) and (9) has been measured from the Ar arc lines. For those stars observed in more than one night, the value given is the resulting resolution for the combined spectrum.

${ }^{a}$ Not in the Cayrel de Strobel et al. (1997) list; magnitude and spectral type from Simbad.

${ }^{\mathrm{b}}$ In Figure 3, this star falls quite off the overall trend of EW with $T_{\text {eff }}$. The spectral type quoted here is from older references. Gray et al. (2006) give it a K9V spectral type (thus moving the point further to the left), but also indicate presence of (minor) chromospheric activity in this star. The spectrum is shown in Figure 1(f).

a bright $(V<13 \mathrm{mag})$ star available in the guide probe patrol field.

The observations were done using the IFU (Allington-Smith et al. 2006, 2007) with the 111 line $\mathrm{mm}^{-1}$ grating, yielding a resolving power of $R \sim 5900$. The list of observations is given in Table 1, where for each object are listed the $V$ magnitude, effective temperature and surface gravity when available, as well as the observing dates and the spectral resolution, measured from the arc lines, for the "blue" (centered at $2.245 \mu \mathrm{m}$ ) and "red" (centered at $2.335 \mu \mathrm{m}$ ) settings, respectively.

The observing conditions also determined the instrument configuration: to achieve $R=5900$ with GNIRS in long slit mode, one would have to use the 0.'30 slit, implying very large slit losses under poor seeing conditions (FWHM $>00^{\prime \prime} 80$ in $K$ ). Given the superior GNIRS IFU performance in the $K$ band (over $90 \%$ of that of the equivalent long slit mode), there was only a small loss in sensitivity by using the IFU+111 line $\mathrm{mm}^{-1}$ grating configuration.
The standard group of observations included a science target, one telluric standard star, a set of calibrations comprising three arcs, and a set of ten QH lamp flats. Calibrations (arcs and flats) were usually observed right after the science target, or after a set of targets was observed, but before the grating was moved to another configuration.

Observing sequences were defined as several (2-5) repeats of ABBA sequences, with a $4^{\prime \prime}$ offset between the A (object) and $\mathrm{B}$ (sky) positions in a direction perpendicular to the long axis of the IFU field-of-view, large enough to move a centered object completely off to sky. On-target efficiency with this setup gets reduced by $50 \%$, but it avoids the problem of overlapping point-spread function wings due to the small size of the IFU if trying to dither on source.

The GNIRS data frames as delivered to the Gemini Science Archive are in the standard Gemini MEF (Multi-Extension Format), where the primary header unit (PHU, extension [0]) includes all header information from telescope, environmental 
monitoring system and instrument, and the data extension [1] contains the pixel values. Data reduction was performed using the tasks in the gemini.gnirs IRAF package, ${ }^{3}$ release Version 1.9, of 2006 July 28 and comprised the following steps:

For the calibrations (flats and arcs):

1. nsprepare: this task reformats the data files to add the IFU Mask Definition File (MDF), which contains the information on the position of the individual slices in detector coordinates, and applies the linearity correction to the pixel data. The resulting file contains the PHU, one binary table extension with the MDF, and one data extension with the actual pixel values.

2. nsreduce: cuts out each of the 21 IFU slices according to the MDF inserted above to a separate data extension. No dark correction was applied to either flats or arcs.

3. nsflat: combines the ten frames by data extension, using ccdclipping for rejection and normalizing by the median of the illuminated area in each slice, as defined in the MDF. On average, the processing resulted in a $\mathrm{S} / \mathrm{N} \sim 200-300$ for each extension, with exception of slices 1 and 21 (which were partly vignetted) and slice 13 (which was damaged).

4. used gemcombine to average the three processed arc frames to improve visibility of faint lines, then nswavelength to obtain the wavelength solution from the combined arc. For the standard Gemini Ar lamp, there were four lines in the "red" setting, and six in the "blue" setting. A low-order polynomial (legendre order $=3$ ) was used for the fitting, with residuals of the order of $0.15 \AA$ or less.

For the science data and telluric stars:

1. nsprepare: same as for the calibrations.

2. nsreduce: after cutting the individual slices to separate data extensions, the task corrects the frames for the flat-field, and subtracts adjacent object-sky frames.

3. nsstack: since we had only one position with actual data (the B position was blank sky), and the targets were all bright point sources observed under poor seeing conditions, we simply stacked all A positions without any effort to improve alignment of the individual frames by shifting according to the offsets registered in the headers. In most cases all frames were within 0 .' 3 tolerance, but there were a few observations where drifts of up to 0.8 were seen, usually due to clouds or very poor seeing affecting the guiding performance.

4. nstransform: the wavelength transformation is applied to the stacked frame.

5. nsextract: finally, we interactively extracted the spectrum from each slice, in order to exclude those with very low signal, since the targets were not always well centered in the IFU field-of-view, the two edge slices, and the damaged slice when the spectrum happened to fall within the damaged region. The output from this task is still an MEF file, with each data extension containing a onedimensional spectrum.

6. used a simple IRAF $\mathrm{cl}$ script wrapped around specred.scombine to combine all valid spectra obtained in the previous step. At this point, a single one-dimensional standard FITS spectrum is created, but most of the information contained in the PHU of the MEF files is lost, as scombine propagates the header of the first extension included in the combining list, ignoring the content of the PHU.

\footnotetext{
3 The Gemini Data Reduction package can be obtained through the Gemini Web pages at http://www.gemini.edu/sciops/data-and-results?q=node/10795.
}

Table 2

NIFS Sample

\begin{tabular}{lcclcc}
\hline \hline \multicolumn{1}{c}{ Object } & Mag $(\mathrm{V})$ & Sp Type & \multicolumn{1}{c}{ Date } & $\lambda_{c}(\mu \mathrm{m})$ & Resolution $(\AA)$ \\
\hline HD 210885 & 7.28 & G8 II & 2007 Oct 15 & 2.25 & 3.17 \\
HD 107467 & 7.37 & G8 III & 2006 Jan 18 & 2.20 & 3.20 \\
HD 105028 & 7.37 & K0 III & 2007 Apr 30 & 2.20 & 3.14 \\
BD +44337 & 8.56 & K5 Ib & 2006 Dec 30 & 2.25 & 3.22 \\
HD 10598 & 8.31 & K2 III & 2006 Dec 30 & 2.25 & 3.22 \\
HD 109655 & 7.07 & K5 III & 2006 Jan 1 & 2.20 & 3.12 \\
HD 3989 & 7.31 & K5 II & 2007 Oct 15 & 2.25 & 3.17 \\
HD 30354 & 8.47 & M2 III & 2007 Jan 30 & 2.25 & 3.26 \\
HD 236791 & 8.91 & M3 III & 2007 Jan 2 & 2.25 & 3.23 \\
HD 27796 & 7.75 & M3 III & 2006 Dec 30 & 2.25 & 3.22 \\
HD 235774 & 8.69 & M5 III & 2007 Oct 15 & 2.25 & 3.17 \\
\hline
\end{tabular}

7. finally, applied the telluric correction in the science data, using the standard specred.telluric task, and combined all spectra for those targets observed in different nights.

8. added back the header information lost in step 6, corresponding to the content of the PHU of the MEF frame obtained in step 5. This preserves all telescope, instrument, and program information.

9. removed the continuum shape by fitting a low-order polynomial to the final one-dimensional spectrum, and corrected all spectra to rest velocity, by measuring the central wavelength of a strong, isolated line ( $\mathrm{Mg}$ I at $2.2814 \mu \mathrm{m})$ and using it as a reference zero point for all data sets. This corrected in a single step the intrinsic radial velocity and any zero-point offset that could exist from the wavelength calibration.

Figures 1(a)-(f) show the resulting spectra, with "red" and "blue" settings combined when it applies.

\subsection{The NIFS Data}

NIFS (McGregor et al. 2003) is an IFU spectrograph with coronographic masks, optimized for use with the Gemini North Adaptive Optics (AO) system Altair. In the $K$ band, the standard NIFS configuration yields a 2 pixel spectral resolution of $R=5290($ at $2.2 \mu \mathrm{m})$, covering the entire 1.99-2.40 $\mu \mathrm{m}$ range in a single setting.

The data were obtained either as program calibrations for GN-2006A-SV-123 and GN-2007A-Q-25, and therefore based solely in observability and brightness, or as part of a "poor weather" program GN-2006B-Q-107, and in this case following the same rationale as the GNIRS sample (bright enough for poor conditions, with proper telluric and guide stars available). The stars observed as part of 06A-SV-123 and 07A-Q-25 used the $\mathrm{AO}$ wavefront sensor for guiding, while for 06B-Q-107, the AO fold was parked and guiding was done using the peripheral wavefront sensor only (no AO correction).

The selected instrument configuration was the K_G5605 grating and HK_G0603 filter, resulting in an FWHM for the arc lamp lines of $3.2 \AA$. Each observation consisted of five individual exposures, with the star centered on the array then offset to each corner. Table 2 lists the observations, where for each object we present the spectral type, observing date(s), spectrum central wavelength (in $\mu \mathrm{m}$ ), and spectral resolution from the arc lines (in $\AA$ ).

The data reduction was done in a similar way as for the GNIRS data, using the corresponding tasks in the gemini.nifs IRAF package. The reduction procedure included trimming 
(a)

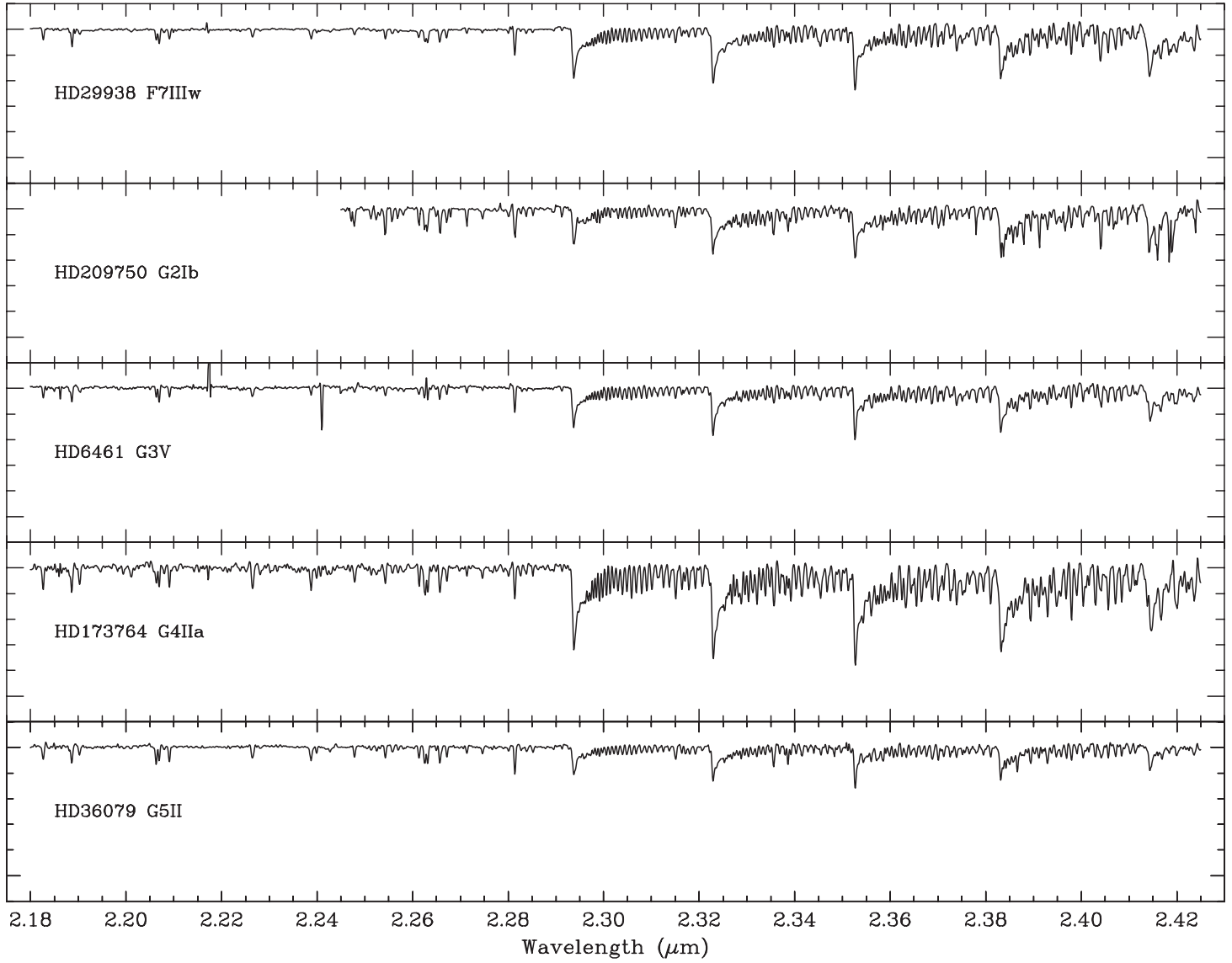

(b)

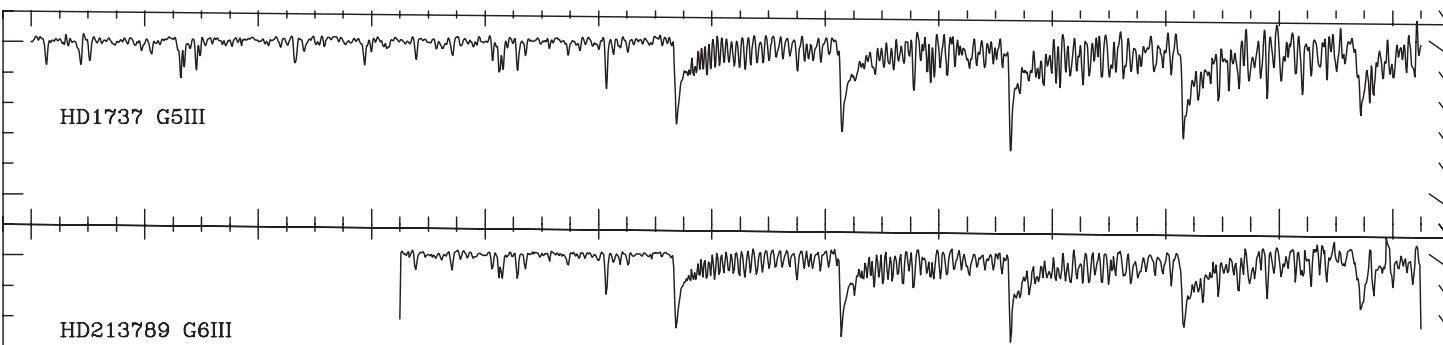

HD213789 G6III

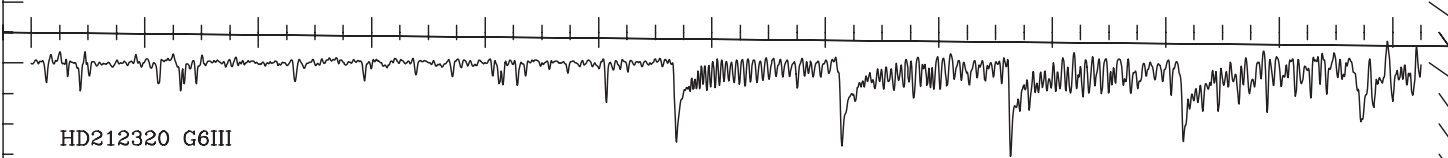

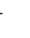

$-$

HD213009 G7III

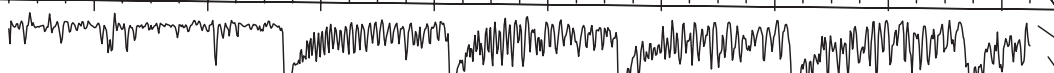

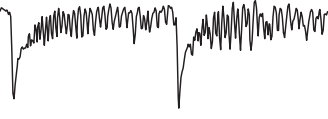

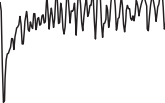

10

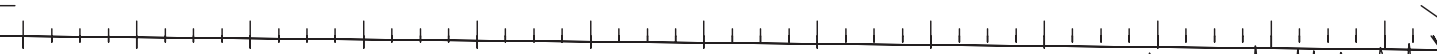

HD35369 G8III

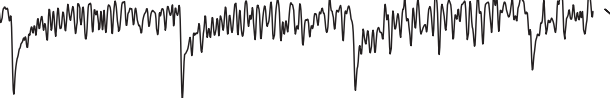

0.5

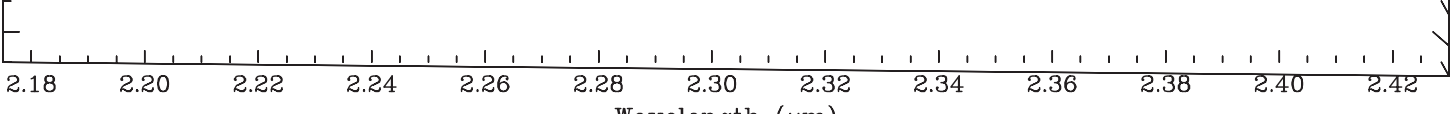

Figure 1. GNIRS template spectra, arranged by spectral type. 
(c)

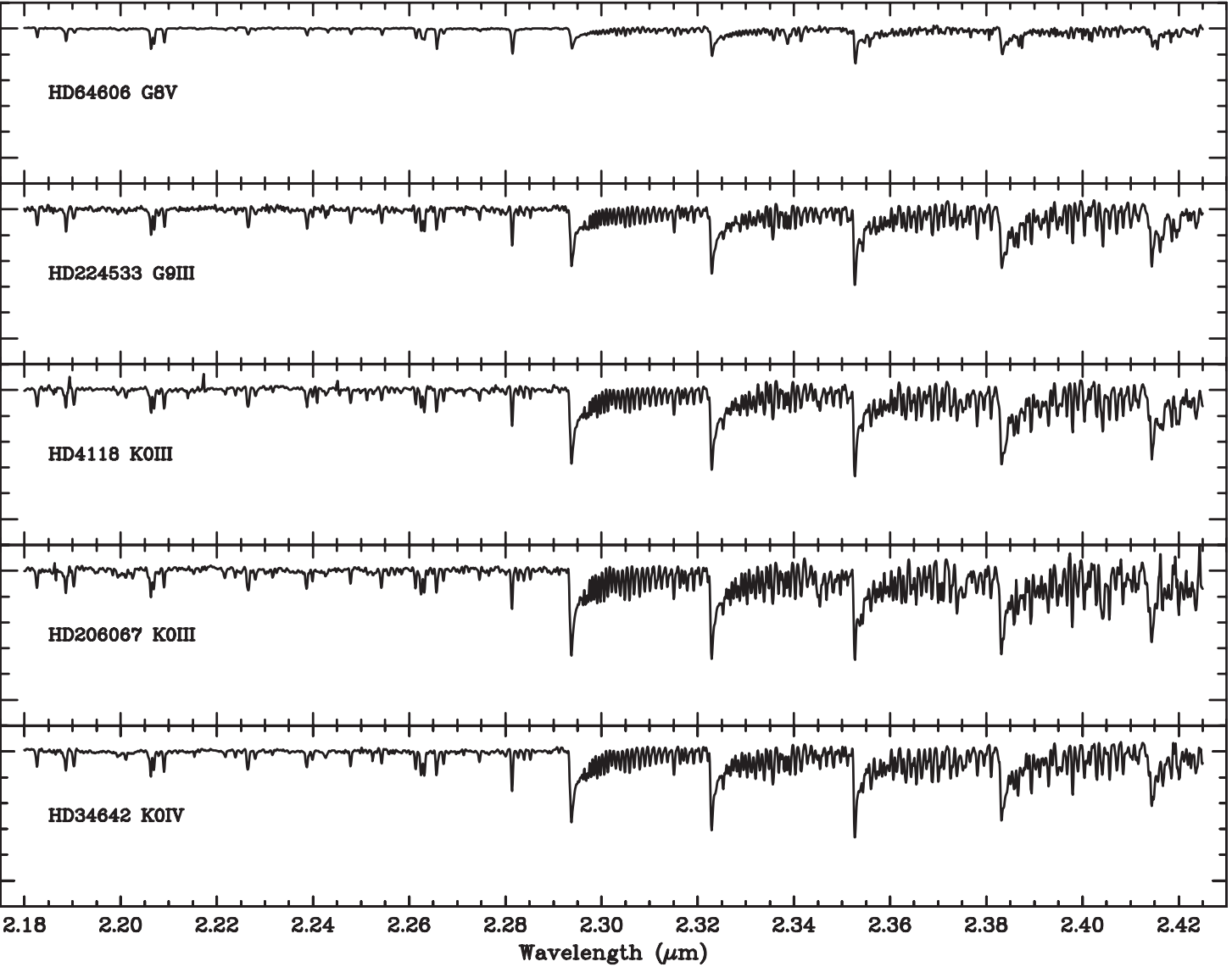

(d)

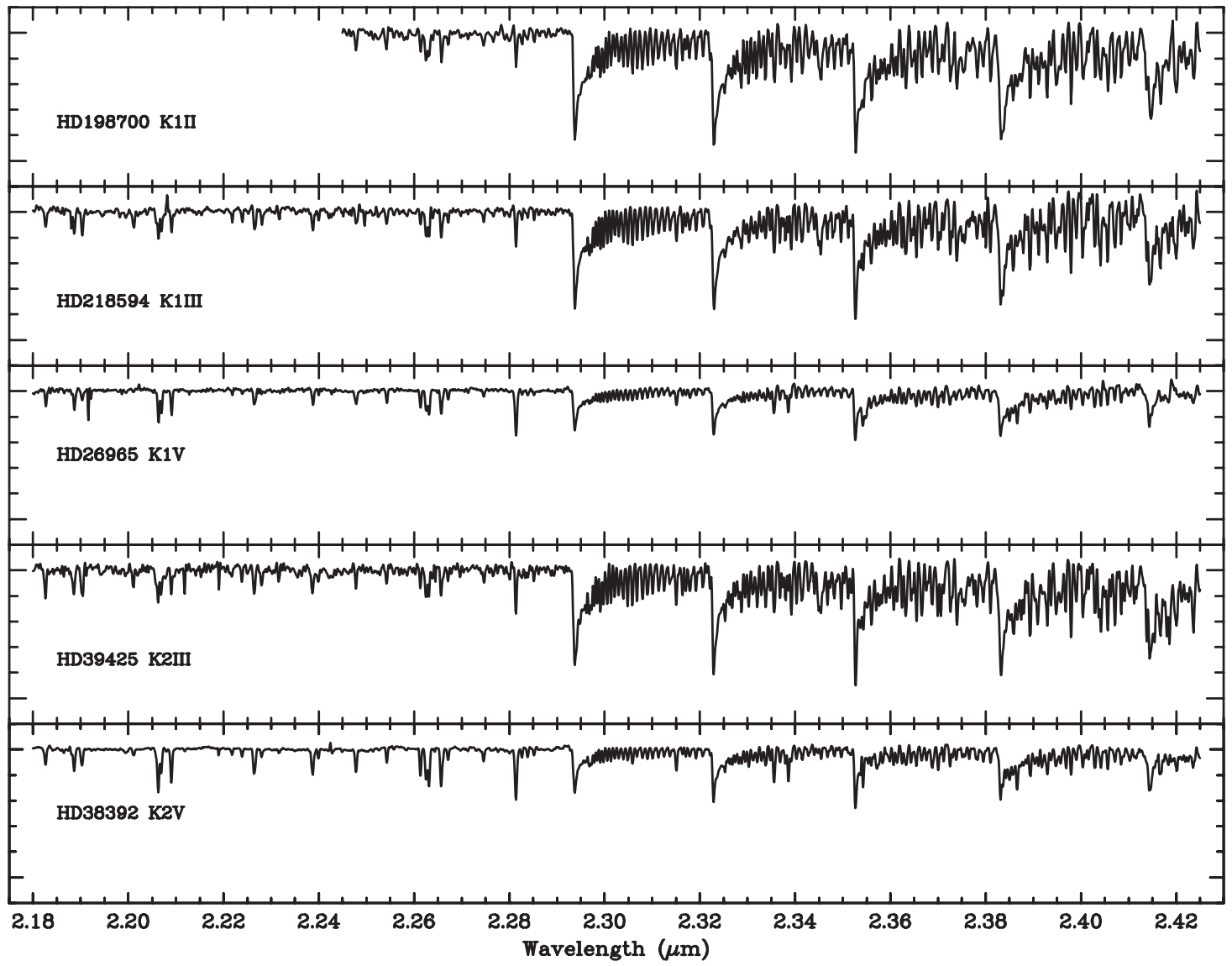

Figure 1. (Continued) 

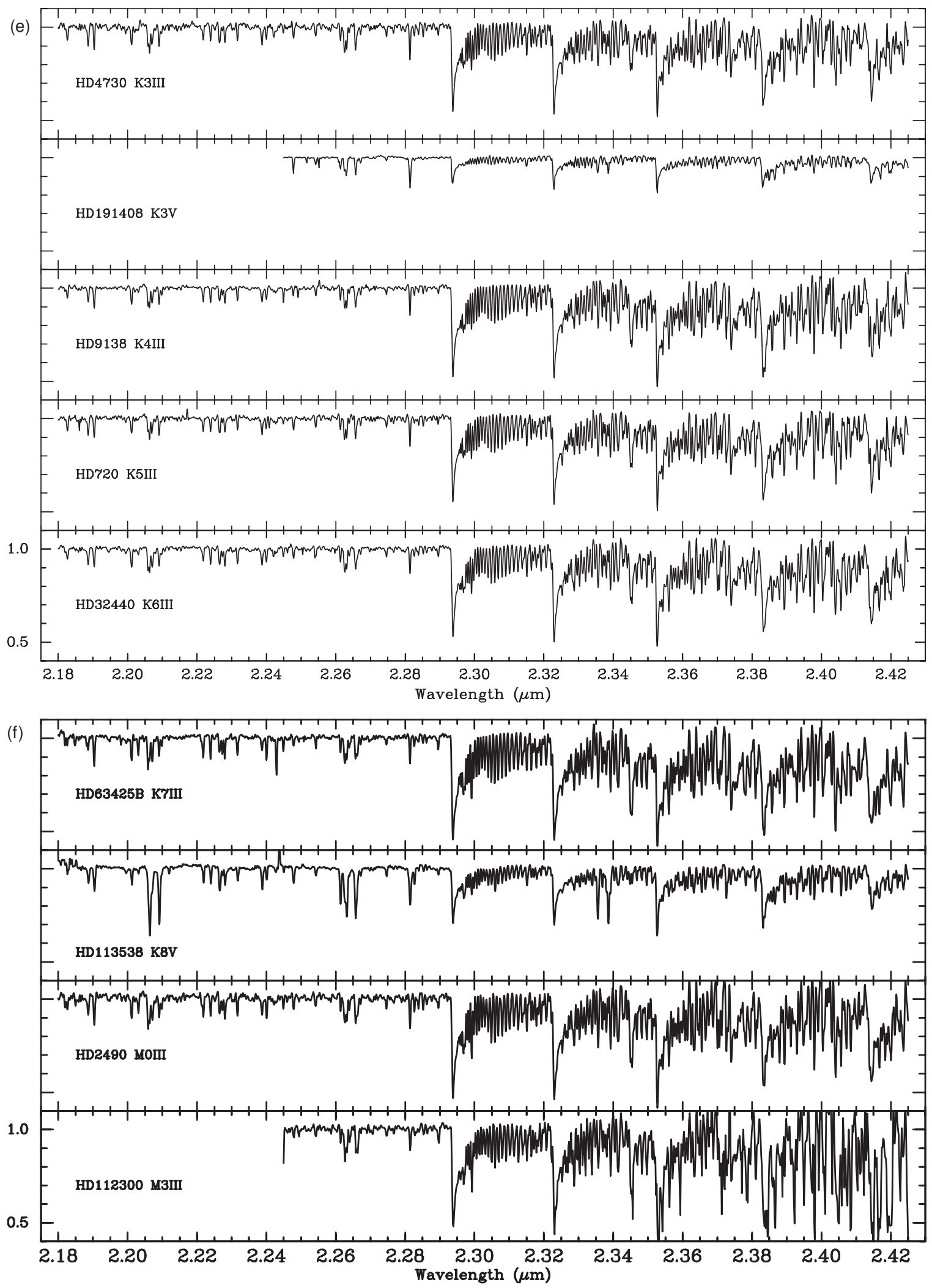

Figure 1. (Continued)

the images, flat-fielding, sky subtraction, wavelength, and s-distortion calibrations. Removal of the telluric bands and flux calibration was executed in a single step by interpolating a black body function to the spectrum of the telluric standard star. Finally, the continuum shape was removed from the spectrum of each star (using the IRAF task continuum), normalizing the continuum fluxes to unity.

Similar to the GNIRS spectra, the extraction procedure results in loss of the primary header content. This was added back to the spectra in order to propagate the relevant instrument/ 
telescope information. The spectra were also shifted to a common reference point using the same Mg I line. Figures 2(a) and (b) show the resulting NIFS spectra.

\section{TEMPLATE FITTING EXAMPLES}

Assuming that a galaxy spectrum is the convolution of a composite stellar spectrum (combination of all the individual stars present in the local stellar population) with the line-of-sight velocity distribution, the most commonly used method to extract the velocity dispersion from the galaxy spectrum employs a cross-correlation technique using one or more stellar spectra as templates (Tonry \& Davis 1979). This technique, however, is sensitive to the effect of template mismatch (e.g., Silge \& Gebhardt 2003; Emsellem et al. 2004; Riffel et al. 2008), with the fitting results being affected by the characteristics of the individual stellar spectrum used as template. To obtain a reliable measurement, one should provide the fitting algorithm with a variety of template stellar spectra, which will be internally attributed different fitting weights to obtain the best result.

Silge \& Gebhardt (2003) (hereafter SG03) have examined the issue and concluded that it is the equivalent width $(\mathrm{EW})$ - or alternatively, the shape - of the $\mathrm{CO}$ band head in the templates that affects the fitting, not the details of the spectral type. Their analysis, using a subsample of stars from the library of Wallace \& Hinkle (1997) (hereafter WH97), showed a trend for the velocity dispersion measured for the galaxy to increase as the EW of the template star's band head increases. They therefore argue that the template sample should span a range of CO EWs, more than simply of spectral types, as the EW of the CO bands is a function of the effective temperature and surface gravity of the star, increasing with decreasing surface gravity or effective temperature.

Figure 3 shows the EW of the first $\mathrm{CO}$ overtone $\left({ }^{12} \mathrm{CO}(2-0)\right.$ at $2.294 \mu \mathrm{m}$ ) in our sample, measured using the IRAF task onedspec.splot in the window 2.293-2.322 $\mu \mathrm{m}$, that is, from the blue edge of the (2-0) band head to the blue edge of the (3-1) band head, plotted as a function of the effective temperature $\left(T_{\text {eff }}\right)$. This window is larger than the one used by SG03 (2.288-2.305 $\mu \mathrm{m})$, but avoids trying to define a "continuum" point among the resolved ${ }^{12} \mathrm{CO}(2-0)$ resonance lines. The resulting EWs are about twice the value obtained using SG03 wavelength range. The EWs in the data ranges from less than 5 to over $30 \AA$. For the template stars not in the sample of Cayrel de Strobel et al. (1997), the value of $T_{\text {eff }}$ was obtained from Table 15.7 of Allen's Astrophysical Quantities, 4th edition (Allen 2000), using a simple linear interpolation between the two nearest spectral types when necessary. Figure 4 presents a sample of spectra in the spectral range including the $\mathrm{Mg}$ I $2.2814 \mu \mathrm{m}$ line (left), the first $\mathrm{CO}$ and part of the second $\left({ }^{12} \mathrm{CO}(3-1)\right.$ at $2.323 \mu \mathrm{m})$ overtones, to illustrate the different EWs and profiles.

As an example of the use of the templates, we selected actual data from three Gemini programs, GS-2005B-Q-65, a GNIRS/IFU+111 line $\mathrm{mm}^{-1}$ observation of the Seyfert galaxy NGC 7582, centered at $2.24 \mu \mathrm{m}$ (Riffel et al. 2009); GN-2006A-SV-123; and GN-2007A-Q-25, NIFS observations of the Seyfert galaxies NGC 4051, and NGC 4258, respectively (Riffel et al. 2008; R. A. Riffel et al. 2010, in preparation). The analysis technique used was the penalized pixel fitting (pPXF) method of Cappellari \& Emsellem (2004), which allows the use of one or several template stellar spectra, and to vary the weights of the contribution of the different templates to obtain the best fit. A complete description of the data, reduction procedure, and analysis is presented in the first two references above.

Figures 5 and 6 present full two-dimensional velocity dispersion maps for NGC 4051 and NGC 7582 respectively, using a single star with smaller (HD 105028) and larger (HD 30354) CO EWs, and the full library. Sample spectra and corresponding fits, extracted from the data cubes, are show in Figure 7. The effect is quite evident: the large-scale structures in the maps are similar for all stars, but the structures in smaller scales and the mean $\sigma$ values vary significantly, with larger EWs in the templates resulting in lower velocity dispersion values measured from the galaxy spectra.

This is the opposite result found by SG03 and prompted a more detailed analysis of the assumed dependency of the fitting result with the stellar template characteristics. To start with, we repeated the fitting of the NGC 4051 off-nucleus spectrum show in Figure 7, using each individual template star in the present library. The galaxy spectrum was then degraded in resolution and the fitting process repeated using each of the stars in the WH97 library with detectable CO absorption (63 objects in total). Since the velocity dispersion of NGC 4051 is quite low, and the resolution of the WH97 templates is $R \sim 3000$, we repeated the same process for the nuclear spectrum of NGC 4258, which has $\sigma \sim 170 \mathrm{~km} \mathrm{~s}^{-1}$, closer to that of the object analyzed by SG03. The results are shown in Figure 8. The fitting results using our templates are shown as filled squares; the open triangles are the results from the fitting using the stars from WH97. An average error of $8 \mathrm{~km} \mathrm{~s}^{-1}$ is assumed (estimated by Riffel et al. 2008 from the fitting of the NGC 4051 datacube). The dotted lines indicate the value of $\sigma$ obtained by fitting the galaxies using all stars from our library simultaneously.

The top panel, with the results for the NGC 4258 nuclear spectrum, gives an indication of why we see a different trend from SG03. Those authors selected only nine stars from the WH97 library, and the dependence found with EW rests strongly in the $\sigma$ value obtained from the template at the lower end of the EW range (see their Figure 4, remembering that the EW plotted here are about a factor of 2 larger due to the different window used in the measurement). The scattering of points for the stars with EW $<10 \AA$ would indicate that, had they chosen another star, they would have perhaps found a very weak to no dependence of $\sigma$ on $\mathrm{EW}$, or even a negative correlation as the NGC 4051 fitting seems to indicate.

For this later galaxy, the low dispersion velocity coupled with the lower resolution of the WH97 create a quite distinct negative correlation, with $\sigma$ decreasing as the $\mathrm{EW}$ increases. Looking at the results using our higher resolution templates, this correlation is much weaker, and essentially rests - just as before-in the one or two templates at the lower end of the EW range. Therefore, the only systematic effect we can derive from our plots is that for templates with lower EW to overestimate the $\sigma$ in the galaxy spectrum when such velocity dispersion is already low and close to the limits allowed by the spectral resolution of the data and templates.

We are, therefore, led to conclude that the resulting velocity dispersion is not directly dependent on the EW of the CO band in the template star, just as SG03 concluded that it was not directly dependent on its spectral type. For NGC 4258, the two K0 III stars in our library result in $\sigma$ values more than $60 \mathrm{~km} \mathrm{~s}^{-1}$ apart, but at the same time it can be seen in the top panel of Figure 8 that templates with essentially the same EW of around $11 \AA$ can yield results ranging from 140 to $240 \mathrm{~km} \mathrm{~s}^{-1}$. 
(a)

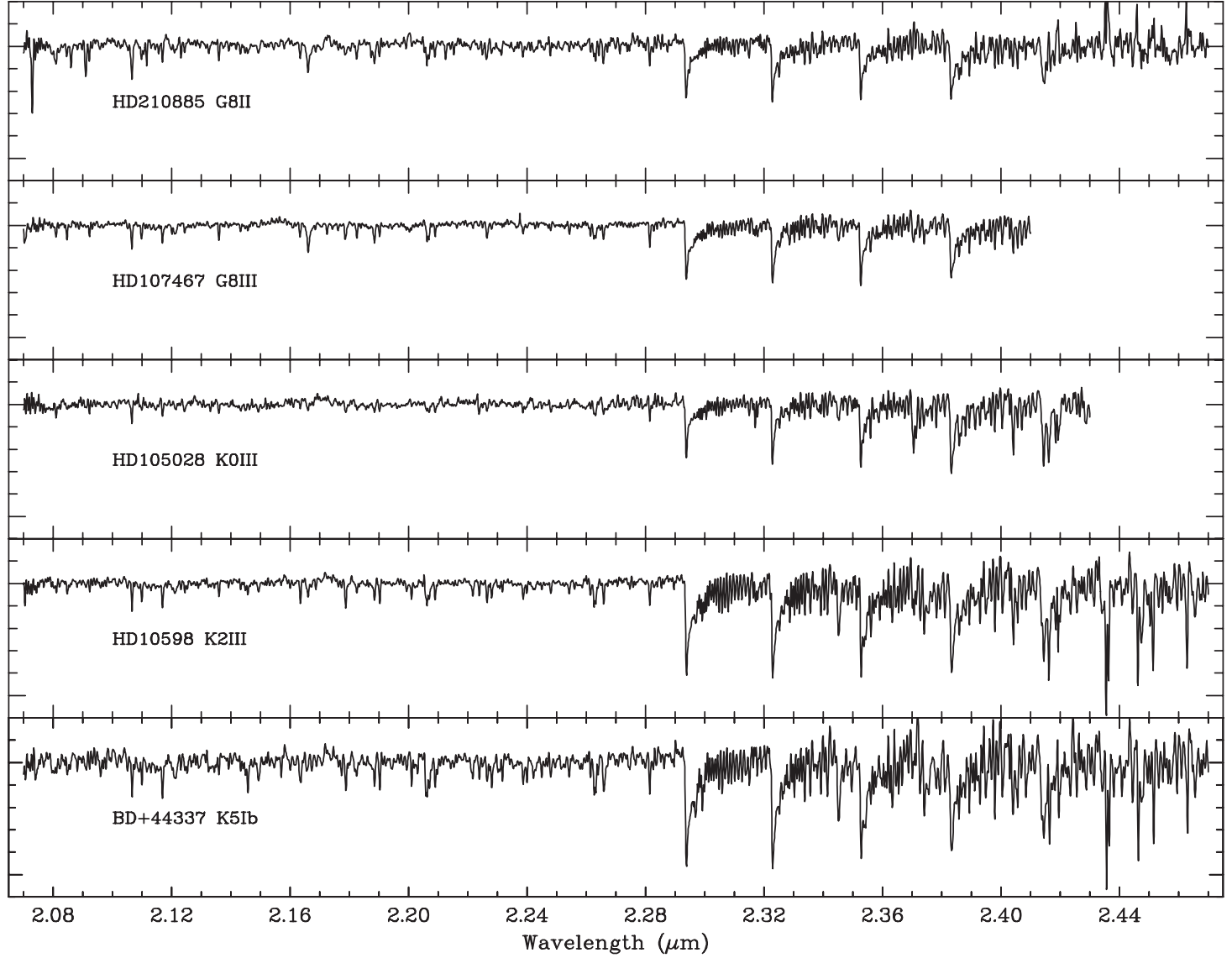

(b)

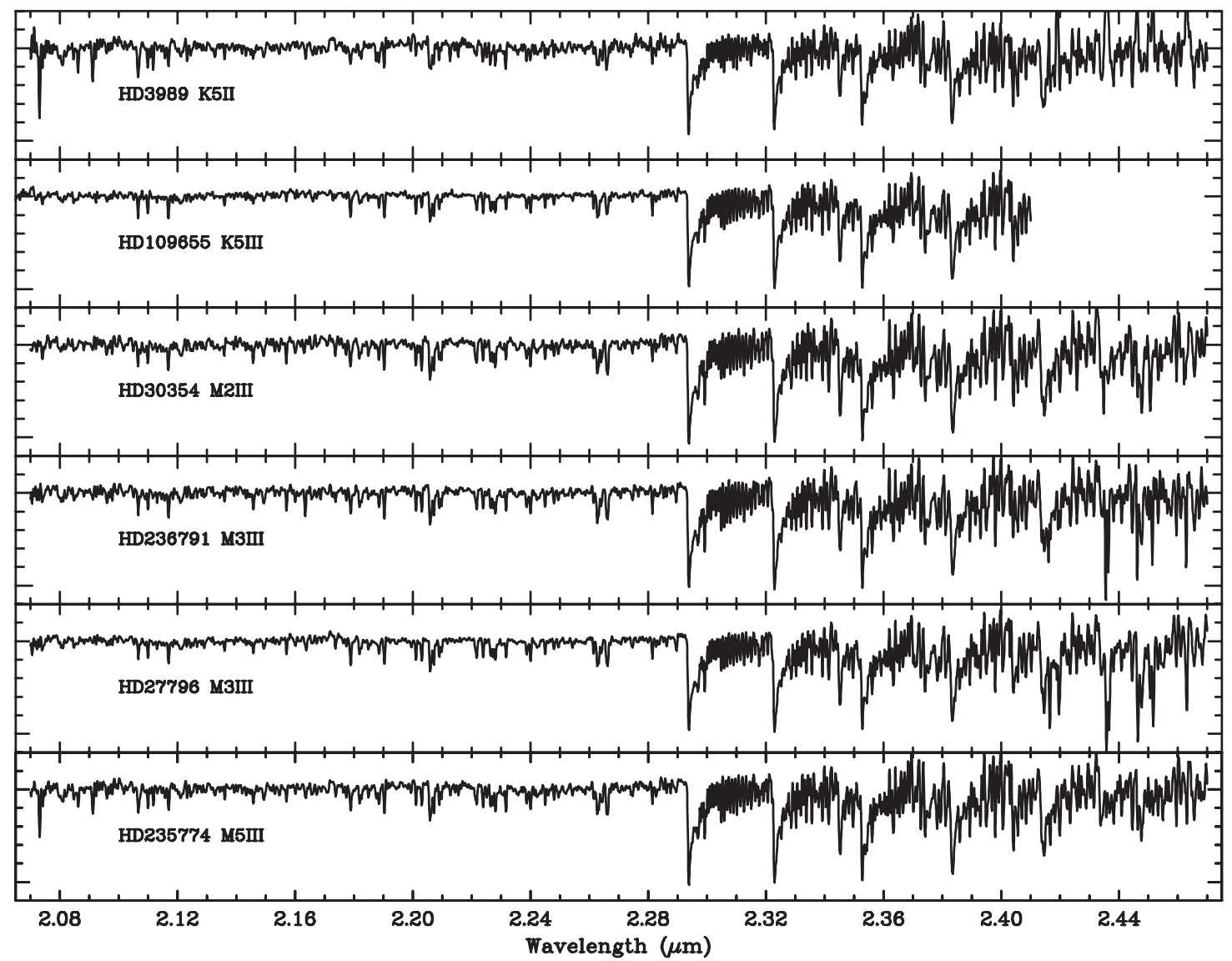

Figure 2. NIFS template spectra, arranged by spectral type. 


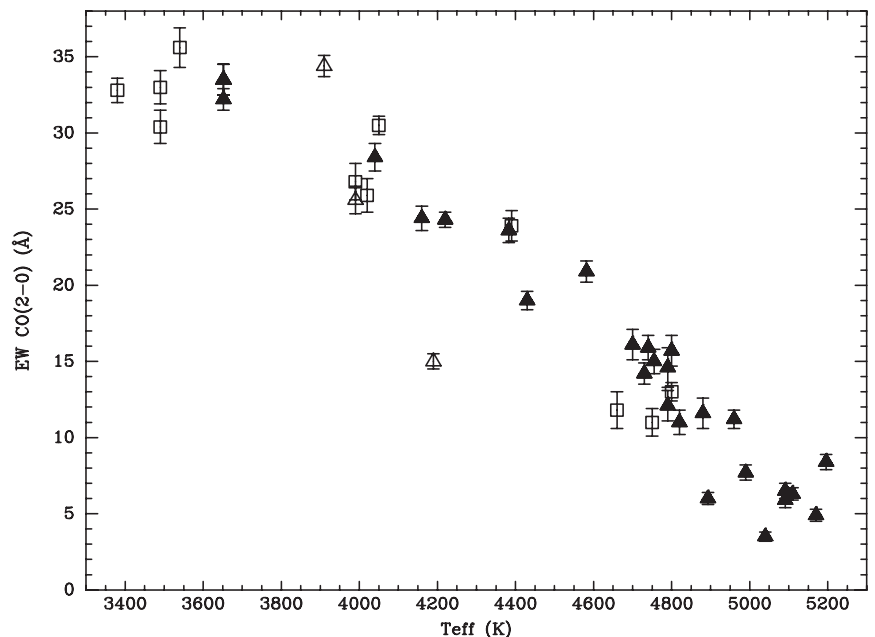

Figure 3. EW of the CO (2-0) $2.294 \mu \mathrm{m}$ band for our sample, plotted as a function of effective temperature. The EW has been measured in the 2.293$2.322 \mu \mathrm{m}$ window and the errors correspond to upper and lower placement of the continuum. The GNIRS templates are represented as triangles, the NIFS sample as squares. Closed symbols are stars with temperatures from Cayrel de Strobel et al. (1997), while open symbols correspond to stars for which the temperature was approximated from the spectral type. The star with $\mathrm{EW}=15 \AA$ and $T_{\text {eff }} \sim 4200 \mathrm{~K}$ is HD 113538 (see note in Table 1).

It is more likely that there is not a simple dependence of the fitting result with any particular characteristic of the template star spectrum, but rather that each template, taken in isolation, yields a result that is better or worse, when comparing with the actual $\sigma$ of the galaxy, depending on how close that individual template is to the overall characteristics of the stellar population contained in the galaxy spectrum. This example illustrates the importance of using a complete stellar library in the determination of the velocity dispersion in galaxies, just as one is needed when analyzing stellar population ages or metallicities in composite spectra.

Figure 9 shows the effect of the slight difference in resolution between the NIFS and GNIRS data. The left panel presents the values of $\sigma$ measured from all the spectra in the NGC 4051 datacube using only the GNIRS stars as templates against the same measurements using only the NIFS stars. There is an excellent correlation between the two values, but with a systematic

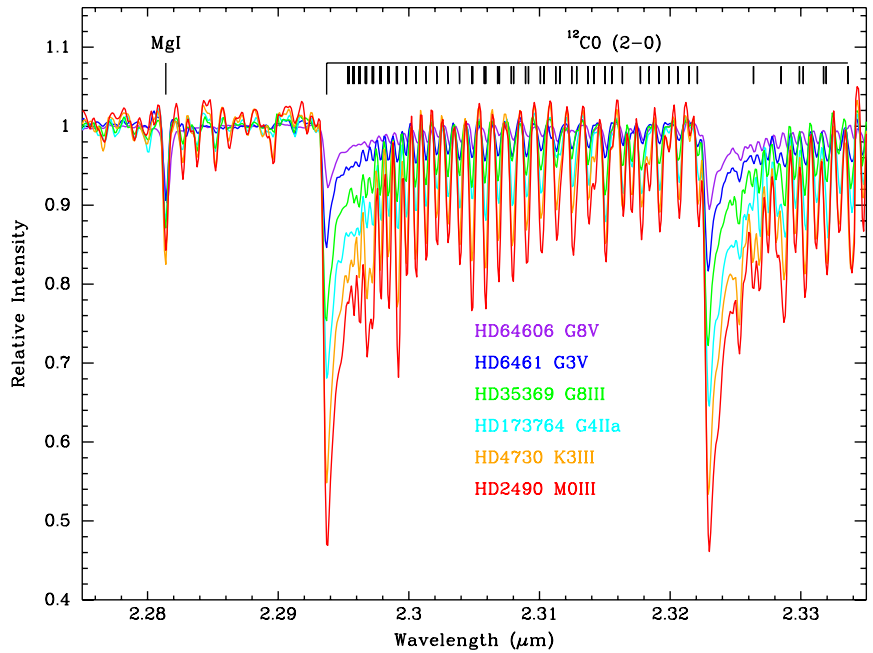

Figure 4. Sample GNIRS template spectra including the $\mathrm{Mg}_{\mathrm{I}}$, the first and part of the second $\mathrm{CO}$ overtones, to illustrate the different EWs and profiles. Note how the individual ${ }^{12} \mathrm{CO}(2-0)$ resonance lines are resolved. The CO line identification was taken from the high resolution atlas of Hinkle et al. (1995).

offset to lower $\sigma$ values using the GNIRS templates. If we consider only the nominal resolving power of the respective gratings, there is a $\sim 5.9 \mathrm{~km} \mathrm{~s}^{-1}$ difference in resolution between the two instruments at $2.3 \mu \mathrm{m}$. The right panel presents the values obtained for $\sigma$ after degrading the resolution of the GNIRS templates to the nominal resolving power of the NIFS grating. The $8 \mathrm{~km}^{-1}$ error bars in the top left corner correspond to the composite error derived by Riffel et al. (2008). The residual tendency for the NIFS data to overestimate the value of $\sigma$ at low values can be traced to the uneven distribution of template characteristics of the two samples (the NIFS templates, being fewer and concentrated towards K5 III types or later, do not by themselves provide a full enough representation of the stellar population of the galaxy).

The individual template spectra also show a range of spectral resolution as measured from the FWHM of the arc lines. The resolution of the NIFS templates ranges from 3.12 to $3.26 \AA$, while that of the GNIRS ones ranges from 2.71 to $3.16 \AA$. When using the templates to fit NIFS and GNIRS data, the difference is
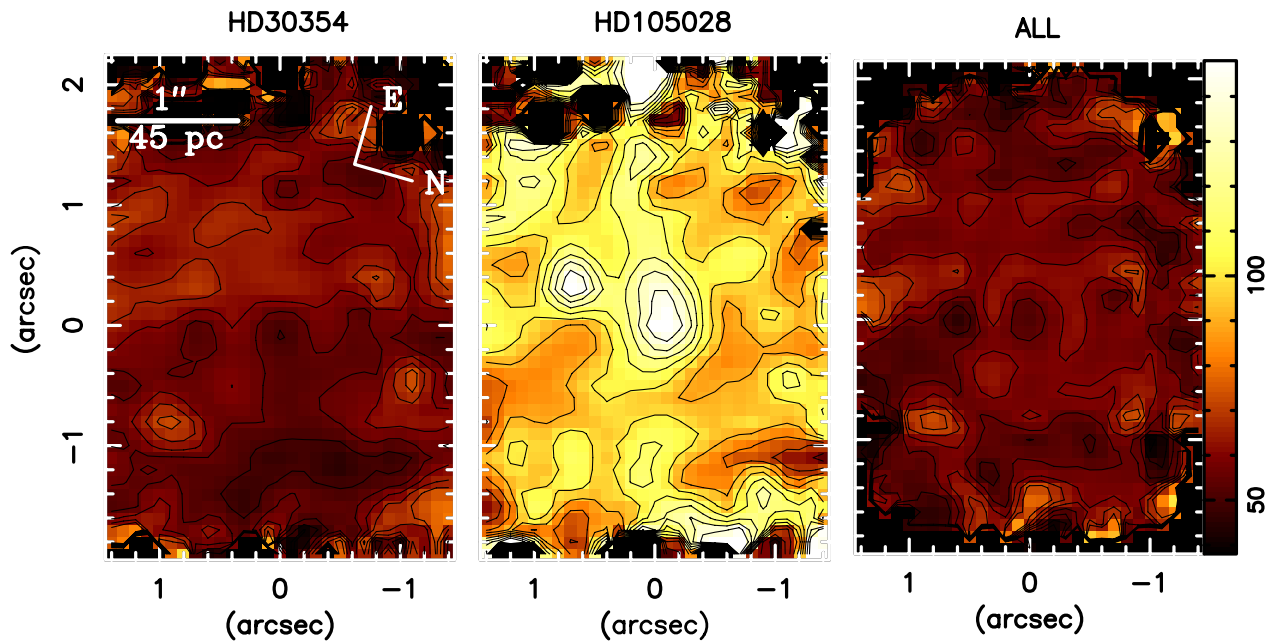

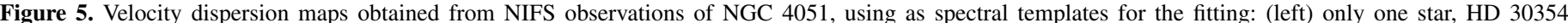

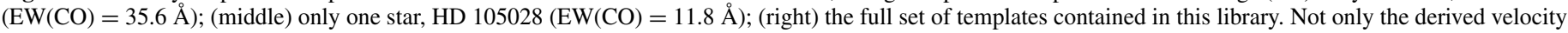
dispersion values, but also some of the structures in small spatial scales are different in each case. 

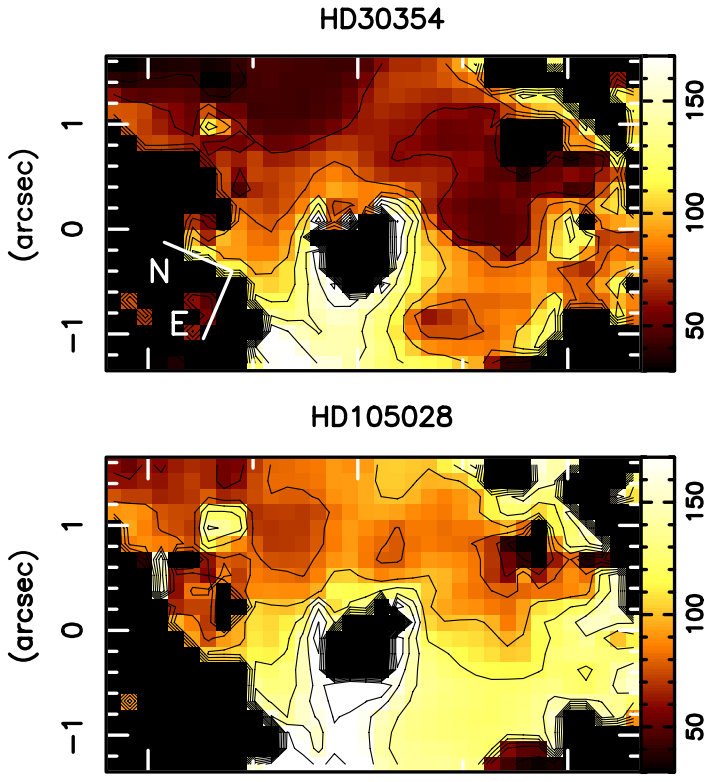

ALL

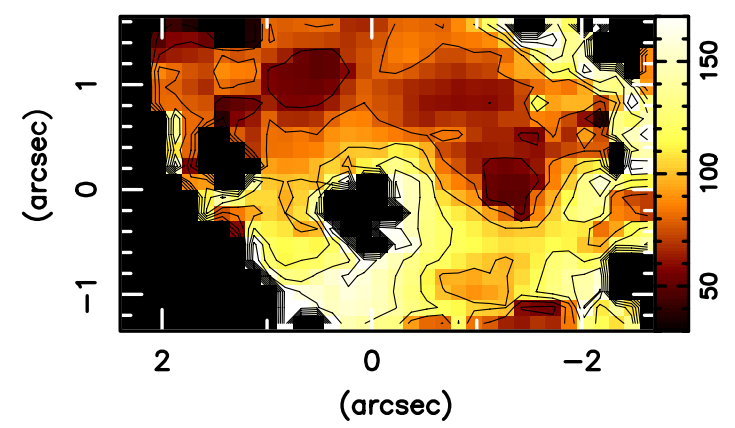

Figure 6. Velocity dispersion maps obtained from GNIRS observations of NGC 7582, using as spectral templates for the fitting: (top) only one star, HD $30354(\mathrm{EW}(\mathrm{CO})=35.6 \AA)$; (middle) only one star, HD $105028(\mathrm{EW}(\mathrm{CO})$ $=11.8 \AA$ ); (bottom) the full set of templates contained in this library. In this case, the overall structure of the three maps is more similar, but the effect of template mismatch in the resulting values for $\sigma$ is quite evident. small enough that this effect gets mixed with the noise and fitting errors, but users that require the best precision should convolve each individual spectrum in the library to match the resolution of the data being fitted. Tables 1 and 2 present the resolution of each stellar template, as measured from the arc lines.

One of the most important results that can be obtained from deriving stellar velocity dispersions in the inner regions of active galactic nuclei is the mass of the central supermassive black hole $\left(M_{\mathrm{BH}}\right)$. The $\left(M_{\mathrm{BH}}\right)$ versus $\sigma$ correlation (Gebhardt et al. 2000; Ferrarese \& Merrit 2000; Tremaine et al. 2002) can be represented as $\log \left(M_{\mathrm{BH}} / M_{\odot}\right)=\alpha+\beta \log \left(\sigma_{*} / \sigma_{0}\right)$, where $\alpha=8.13 \pm 0.06, \beta=4.02 \pm 0.32$, and $\sigma_{0}=200 \mathrm{~km} \mathrm{~s}^{-1}$. If we take the measurements presented in Figure 5, the value obtained for the bulge central velocity dispersion of the galaxy NGC 4051 using the full library for the fitting is $\sim 60 \mathrm{~km} \mathrm{~s}^{-1}$, which from the above equation results in $M_{\mathrm{BH}} \sim 1.1 \times 10^{6} M_{\odot}$. On the other hand, if only the star HD 105028 (a K0III, the spectral type most commonly used as template) was available for this analysis, the result would be a velocity dispersion of $\sim 105 \mathrm{~km} \mathrm{~s}^{-1}$, and thus $M_{\mathrm{BH}} \sim 1 \times 10^{7} M_{\odot}$, a full order of magnitude difference!

It is not directly obtainable from the analysis done here if the effect of using the same single stellar template to determine the velocity dispersion in a sample of objects will be a systematic over- or under-evaluation of $\sigma$. It may be an effect proportional to the actual value of the velocity dispersion, or even a pure scattering of the points around the actual values, depending on how close a representation of the local stellar population is to the chosen stellar template. In any case, if the mass of the supermassive black hole for a certain object or sample of objects is determined by other methods, while the value of $\sigma$ is measured by template fitting, the effect of template mismatch can introduce further scattering on the data points in the $M_{\mathrm{BH}}-\sigma$ correlation.

It is, in any case, quite evident that the improvements in the quality of the data sets and in the analysis methods have led studies of stellar velocity dispersion fitting in external galaxies to join the ranks of the more general issue of composite stellar population analysis, in the sense that a well-known, as complete as possible, template base is needed.
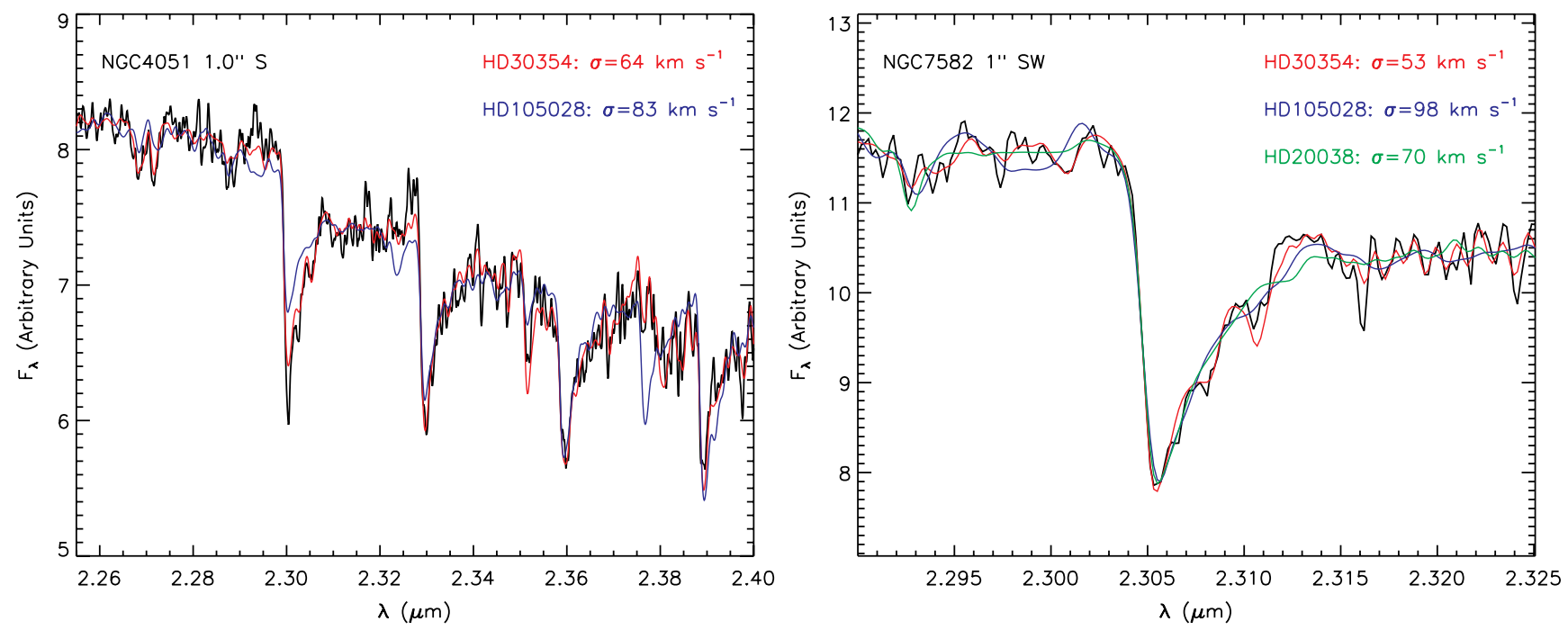

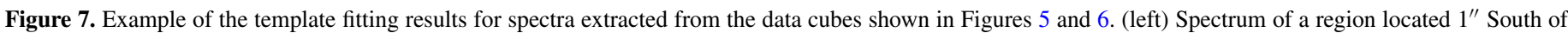

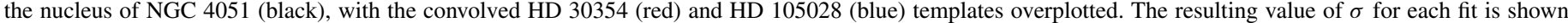

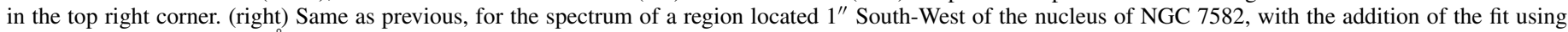
HD $20038(\mathrm{EW}(\mathrm{CO})=8.4 \AA)$ as template (green $)$. 


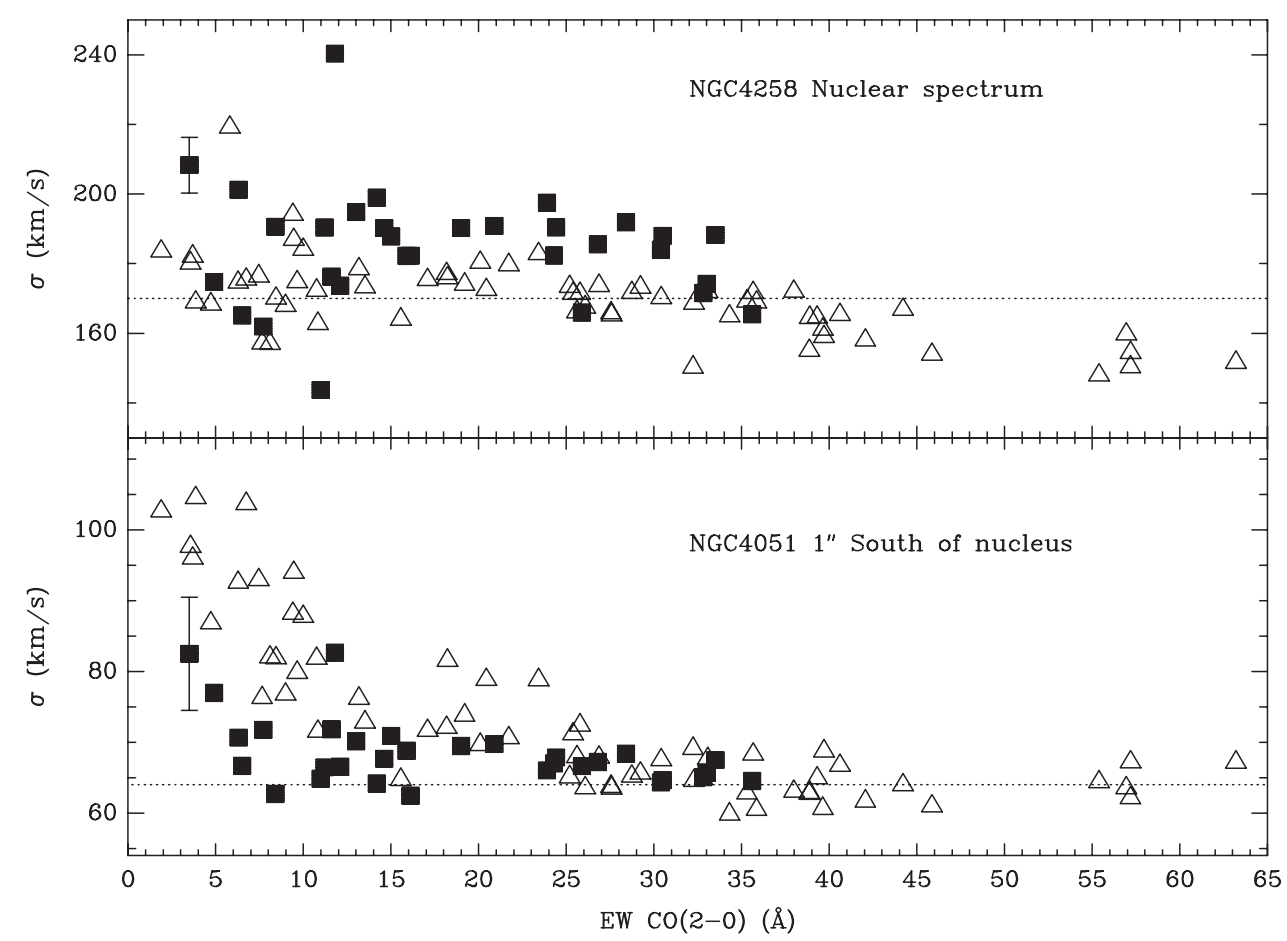

Figure 8. Velocity dispersion resulting from fitting individual template stars from this library (filled squares) and that of Wallace \& Hinkle (1997) (open triangles), plotted as a function of the EW of the ${ }^{12} \mathrm{CO}(2-0)$ absorption band. The top panel presents the results for the nuclear spectrum of NGC 4258 , the bottom panel for an off-nuclear spectrum in NGC 4051. The dashed line is the velocity dispersion value found by using all the stars in the present library as initial input to the fitting program. The error bar corresponds to an average error of $8 \mathrm{~km} \mathrm{~s}^{-1}$; individual errors tend to be slightly larger $\left(10-12 \mathrm{~km} \mathrm{~s}{ }^{-1}\right)$ for low EW templates fitting a low $\sigma$ spectrum such as NGC 4051, or slightly smaller for larger template EW and $\sigma$.
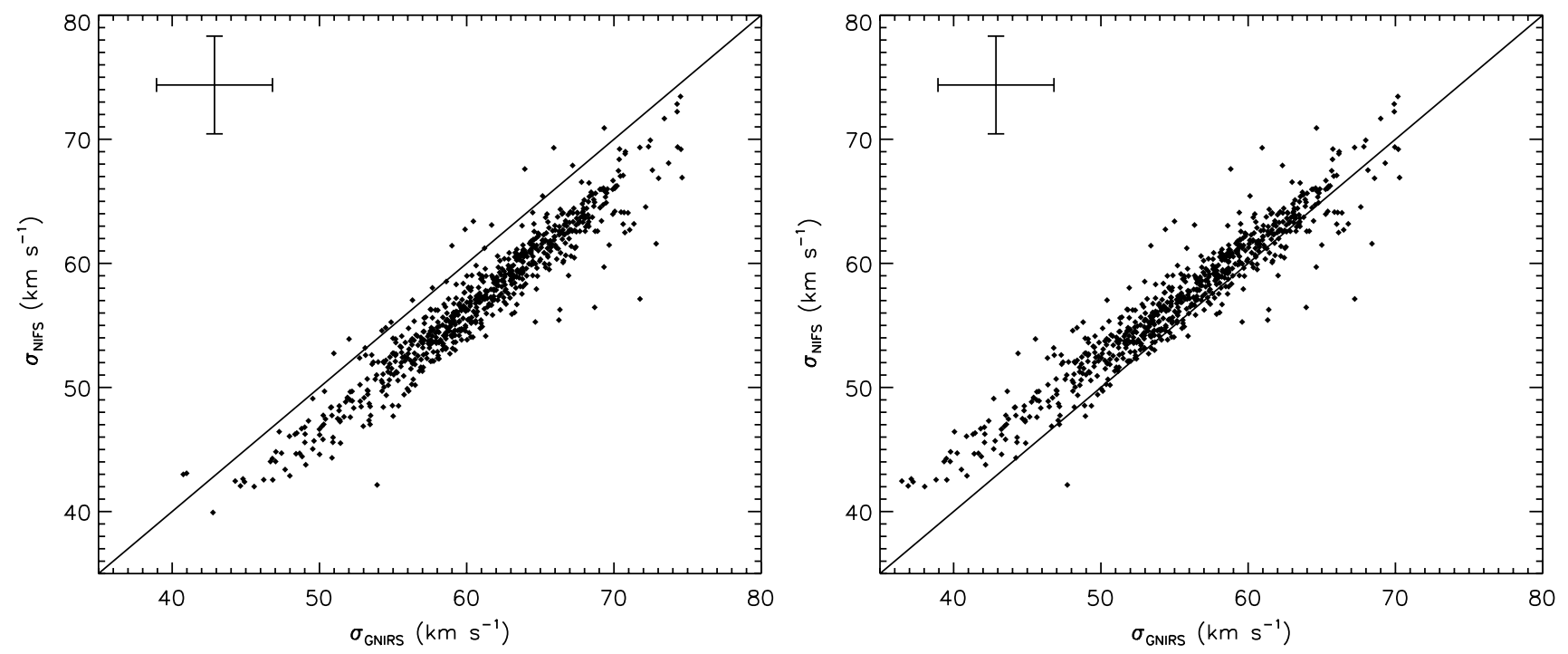

Figure 9. Correlation between the velocity dispersion measured from the NGC 4051 datacube using only the stellar templates from the GNIRS sample [ $\sigma$ (GNIRS)] and from the NIFS sample $[\sigma$ (NIFS)]. The left panel shows the result using the templates in their native resolution, while in the right panel the resolution of the GNIRS templates was degraded to match the nominal resolution of the NIFS grating.

\section{AVAILABILITY OF THE LIBRARY}

The Gemini library of late-type stellar templates can be downloaded from the Gemini Web site as individual objects or in two compressed files for the NIFS and GNIRS stars: http://www.gemini.edu/sciops/instruments/nearir-resources/? $\mathrm{q}=$ node $/ 10167$.

The page contains links to the final processed spectra and details of the data reduction. It also presents the history and release notes for the different versions of the library. The version described in this paper corresponds to V1.5 from 2009 January 31 .

The data are presented in standard FITS format, and the user can select either the GNIRS "red" (2.24-2.42 $\mu \mathrm{m})$ or "blue" $(2.15-2.32 \mu \mathrm{m})$ spectral ranges at their native spectral binning or the combined spectrum (when both ranges were observed), rebinned to $1 \AA$ pixel $^{-1}$. The NIFS spectra are also presented at native binning and rebinned to $1 \AA$ pixel $^{-1}$. All data were kept 
with their original spectral resolution, so the users can more adequately adjust the templates to the actual resolution of their own data sets.

The community at large is welcome to download all or part of the library as needed, and users of GNIRS and NIFS are encouraged to explore its use as an alternative to requesting further observation of spectral standards with their science programs. All the GNIRS data collected under program GS-2006B-DD-3 has been made public from the start in the Gemini Science Archive. The NIFS data were subject to the standard proprietary period. If the raw data are re-processed to be used in papers or publications, please use the standard Gemini acknowledgment text for archival data and the appropriate program IDs.

The authors thank the Gemini Deputy Director and Head of Science, Jean-Rene Roy, and the former Gemini South Head of Science Operations, Michael West, for the support and time allocation for our GNIRS program. Many thanks as well to all the Gemini South observers and SSAs that so positively believed that no conditions were ever too poor to give GS-2006B-DD-3 a chance!

We thank the anonymous referee for pointing out the issue that led to the more thorough discussion of the velocity dispersion dependency with template characteristics, which significantly enhanced the scope of this paper.

Based on observations obtained at the Gemini Observatory, which is operated by the Association of Universities for Research in Astronomy, Inc., under a cooperative agreement with the NSF on behalf of the Gemini partnership: the National Science Foundation (United States), the Science and Technology Facilities Council (United Kingdom), the National Research Council (Canada), CONICYT (Chile), the Australian Research Council (Australia), Ministério da Ciencia e Tecnologia (Brazil), and Ministerio de Ciencia, Tecnología e Innovación Productiva (Argentina). Data taken under program IDs: GS-2006B-DD-3, GN-2006A-SV-123, GN-2006B-Q-107, and GN-2007A-Q-25.

Facilities: Gemini:South, Gemini:Gillett.

\section{REFERENCES}

Allington-Smith, J. R., Content, R., Dubbeldam, C. M., Robertson, D. J., \& Preuss, W. 2006, MNRAS, 371, 380

Allington-Smith, J., et al. 2007, MNRAS, 376, 785

Barbosa, F. K. B., Storchi-Bergmann, T., Cid Fernandes, R., Winge, C., \& Schmitt, H. 2006, MNRAS, 371, 170

Cappellari, M., \& Emsellem, E. 2004, PASP, 116, 138

Cappellari, M., Neumayer, N., Reunanen, J., van der Werf, P. P., de Zeeuw, P. T., \& Rix, H.-W. 2009, MNRAS, 394, 660

Cappellari, M., et al. 2007, MNRAS, 379, 418

Cayrel de Strobel, G., Soubiran, C., Friel, E. D., Ralite, N., \& Francois, P. 1997, A\&AS, 124, 299

Cox, A. (ed.) 2000, Allen's Astrophysical Quantities (4th ed.; New York: Springer)

Dumas, G., Mundell, C. G., Emsellem, E., \& Nagar, N. M. 2007, MNRAS, 379 , 1249

Elias, J. H., et al. 1998, Proc. SPIE, 3354, 555

Emsellem, E., Greusard, D., Combes, F., Friedli, D., Leon, S., Pécontal, E., \& Wozniak, H. 2001, A\&A, 368, 52

Emsellem, E., et al. 2004, MNRAS, 352, 721

Ferrarese, L., \& Merrit, D. 2000, ApJ, 539, L9

Förster Schreiber, N. M. 2000, AJ, 120, 2089

Ganda, K., Falcón-Barroso, J., Peletier, R. F., Cappellari, M., Emsellem, E., McDermid, R. M., de Zeeuw, P. T., \& Carollo, C. M. 2006, MNRAS, 367, 46

Gebhardt, K., et al. 2000, ApJ, 539, L13

Gray, R. O., Corbally, C. J., Garrison, R. F., McFadden, M. T., Bubar, E. J., McGahee, C. E., O’Donoghue, A. A., \& Knox, E. R. 2006, AJ, 132, 161

Hinkle, K., Wallace, L., \& Livingston, W. 1995, PASP, 107, 1042

Ivanov, V. D., Rieke, M. J., Engelbracht, C. W., Alonso-Herrero, A., Rieke, G. H., \& Luhman, K. L. 2004, ApJS, 151, 387

Kleinmann, S. G., \& Hall, D. N. B. 1986, ApJS, 62, 501

Márquez, I., Masegosa, J., Durret, F., González Delgado, R. M., Moles, M., Maza, J., P'erez, E., \& Roth, M. 2003, A\&A, 409, 459

McGregor, P. J., et al. 2003, Proc. SPIE, 4841, 1581

Ramirez, S. V., Depoy, D. L., Frogel, J. A., Sellgren, K., \& Blum, R. D. 1997, AJ, 113,1411

Riffel, R. A., Storchi-Bergmann, T., Dors, O. L., \& Winge, C. 2009, MNRAS, 393, 783

Riffel, R. A., Storchi-Bergmann, T., Winge, C., McGregor, P. J., Beck, T., \& Schmitt, H. 2008, MNRAS, 385, 1129

Silge, J., \& Gebhardt, K. 2003, AJ, 125, 2809

Tonry, J., \& Davis, M. 1979, AJ, 84, 1511

Tremaine, S., et al. 2002, ApJ, 574, 740

Wallace, L., \& Hinkle, K. 1997, ApJS, 111, 445 